\section{JTI}

JOURNAL OF

TRAUMA AND INJURY

\title{
Experiences of Emergency Surgical Treatment for a COVID-19 Patient with Severe Traumatic Brain Injury at a Re- gional Trauma Center: A Case Report
}

\author{
Jung-Ho Yun, M.D. \\ Department of Neurological Surgery, College of Medicine, Dankook University, Cheonan, \\ Korea
}

Received: September 9, 2021

Revised: September 19, 2021

Accepted: September 23, 2021

\section{Correspondence to}

Jung-Ho Yun, M.D.

Department of Neurological Surgery, Dankook University Hospital, 201

Manghyang-ro, Dongnam-gu, Cheonan 31116, Korea

Tel: +82-41-550-3921

Fax: +82-41-550-6280

E-mail: tjburk@hanmail.net

ORCID: https://orcid.org/0000-00034091-1346

Various medical scenarios have arisen with the prolonged coronavirus disease 2019 (COVID-19) pandemic. In particular, the increasing number of asymptomatic COVID-19 patients has prompted reports of emergency surgical experiences with these patients at regional trauma centers. In this report, we describe an example. A 25-yearold male was admitted to the emergency room after a traffic accident. The patient presented with stuporous mentality, and his vital signs were in the normal range. Lacerations were observed in the left eyebrow area and preauricular area, with hemotympanum in the right ear. Brain computed tomography showed a contusional hemorrhage in the right frontal area and an epidural hematoma in the right temporal area with a compound, comminuted fracture and depressed skull bone. Surgical treatment was planned, and the patient was intubated to prepare for surgery. A blood transfusion was prepared, and a central venous catheter was secured. The initial COVID-19 test administered upon presentation to the emergency room had a positive result, and a confirmatory polymerase chain reaction (PCR) test was administered. The PCR test confirmed a positive result. Emergency surgical treatment was performed because the patient's consciousness gradually deteriorated. The risk of infection was high due to the open and unclean wounds in the skull and brain. We prepared and divided the COVID-19 surgical team, including the patient's transportation team, anesthesia team, and surgical preparation team, for successful surgery without any transmission or morbidity. The patient recovered consciousness after the operation, received close monitoring, and did not show any deterioration due to COVID-19.

Keywords: COVID-19; Brain injuries, traumatic

This is an Open Access article distributed under the terms of the Creative Commons Attribution Non-Commercial License (http://creativecommons.org/licenses/by-nc/4.0/) which permits unrestricted noncommercial use, distribution, and reproduction in any medium, provided the original work is properly cited. 


\section{INTRODUCTION}

In December 2019, a pneumonia caused by unknown origins and factors was discovered in Wuhan, Hubei province in China. The disease was later referred to as corona virus disease 2019 (COVID-19) [1,2]. A respiratory illness that was caused by the coronavirus, 2019-novel coronavirus ( $\mathrm{nCoV}$ ), was shown to be transmitted by contact with infectious materials and was characterized especially by high fever, cough, and respiratory stress that may evolve to pneumonia and respiratory failure. As the coronavirus pandemic has spread worldwide, many countries have gone beyond the stage of protection and prevention of infections and are now moving on to the stage of developing corona-based strategies for after vaccination. In this situation, quarantine practices are very important in hospitals, especially in trauma centers where emergency patients are commonly treated. Here, we present experiences with surgical treatment at Dankook University Hospital Regional Trauma Center when emergency surgery is unavoidable for traumatic brain injury patients with a confirmed asymptomatic coronavirus infection.

\section{CASE REPORT}

A 25-year-old male was admitted to the emergency room after a traffic accident. The patient was stuporous mentally, and his vital signs were in the normal range. Lacerations were observed in the left eyebrow area and preauricular area, with a hemotympanum in the right ear.
Brain computed tomography (CT) showed a contusional hemorrhage in the right frontal area and epidural hematoma in the right temporal area with a compound, comminuted fracture and depressed skull bone (Fig. 1A, B). Surgical treatment was planned, and the patient was intubated in preparation for surgery. Blood transfusion preparation was completed, and a central venous catheter was secured. The patient received an emergency coronavirus test immediately in the emergency medical center, and the test showed a positive result, which was then re-examined via a polymerase chain reaction (PCR) test. The patient was immediately quarantined after confirming the result, and all the contacted medical staff were tested for COVID-19. Fortunately, the patients had no symptoms related to COVID-19 disease, and there were no specific abnormalities in the results of the initial chest X-ray and chest CT scan (Fig. 1C, D). After 3 hours of patient isolation, the patient was subjected to a brain CT scan after wearing protective gear due to decreased consciousness and decreased muscle strength in the left upper limb. As indicated by a brain CT scan, the frontal contusional hemorrhage increased intracerebral hemorrhage and spread to an intraventricular hemorrhage (Fig. 2). The patient's pupil response was in the normal range after injecting mannitol to control intracranial pressure while waiting for the final COVID-19 PCR test results. After 1 hour, his final COVID-19 PCR test yielded a positive result. We decided to perform emergency surgical treatment because the patient's consciousness was gradually deteriorating and there was a high risk of infection due to the open unclean wounds to the skull and brain. We
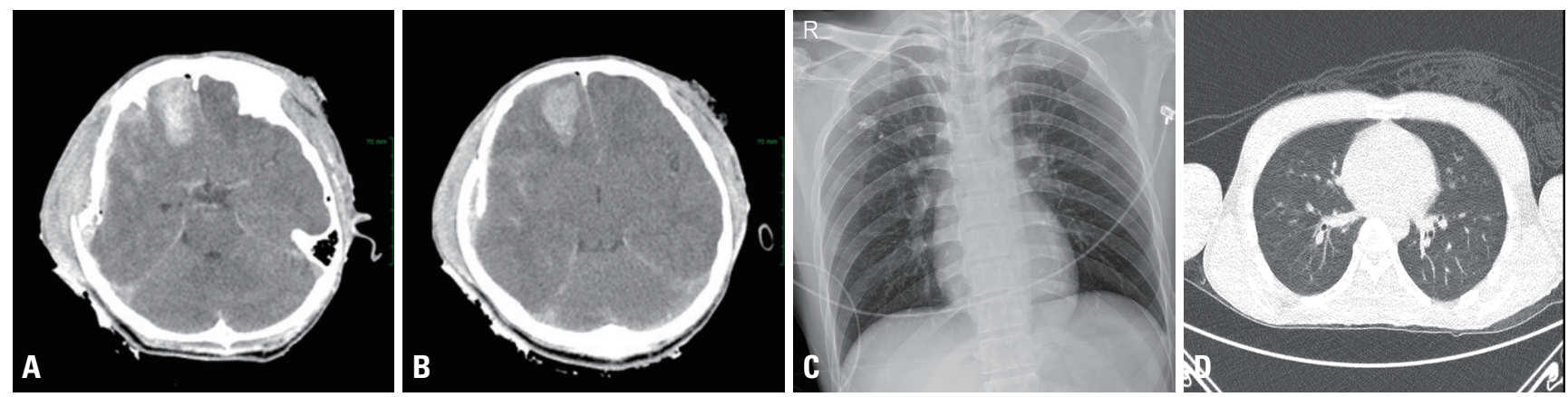

Fig. 1. Initial radiologic evaluation. Brain CT axial images reveal epidural hemorrhaging in the right temporal lesion with a comminuted, (A) depressed skull fracture and (B) traumatic contusion in the right frontal lobe. (C) Initial chest AP and (D) chest CT scan showed no specific lesion. CT: computed tomography, AP: anteriorposterior. 


\section{JTI}

then prepared and divided the "COVID surgical team" in Dankook University Hospital Regional Trauma Center, including the patient's transportation team, anesthesia team, and surgical preparation team for successful surgery without re-infection or morbidity.

The surgery was performed by one trauma neurosurgeon, and one senior resident participated as an operative assistant. Operative finding showed a large amount of intracerebral hematoma in the right frontal area with
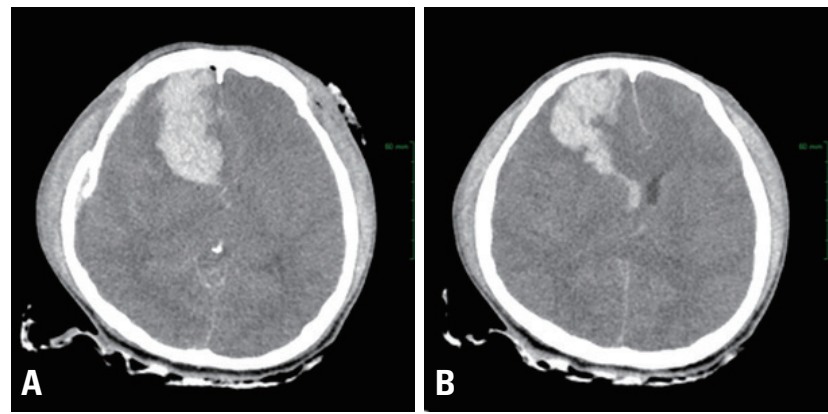

Fig. 2. (A, B) Follow- up brain CT axial image revealed a large hematoma in the right frontal area. CT: computed tomography. moderate cerebral edema and the bleeding originating from the rupture of a blood vessel on the brain surface. We performed a craniectomy with duraplasty and hematoma evacuation using suction and a brain needle, and complete hematoma removal was achieved. The total operation time was 2 hours and 10 minutes, including all anesthesia times, and the actual blood loss was approximately $500 \mathrm{~mL}$. Three packs of PRC were supplied for
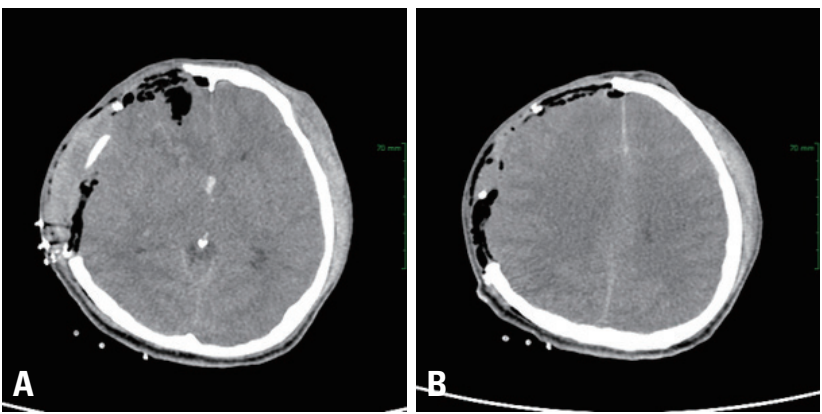

Fig. 3. (A, B) Postoperative brain CT scan demonstrating nearly complete removal of the large hematoma in the right frontal and temporal area. CT: computed tomography.

Table 1. The European Society of Emergency Surgery board endorsed these recommendations

\begin{tabular}{|c|c|c|}
\hline Step & Title & Main subject \\
\hline 1 & General recommendation for surgical services & $\begin{array}{l}\text { Trauma and emergency general surgery cases should proceed to the OR in a timely } \\
\text { manner with consideration to COVID-19 guidelines for symptomatic patient. }\end{array}$ \\
\hline 2 & $\begin{array}{l}\text { Emergency surgery for critically ill COVID-19 } \\
\text { positive or suspected patients-preoperative } \\
\text { planning }\end{array}$ & $\begin{array}{l}\text { Trauma cases should be excluded from discussions regarding goals of care only if } \\
\text { immediate surgery is required. Any advanced directives should be respected and re- } \\
\text { viewed. }\end{array}$ \\
\hline 3 & OR setup & $\begin{array}{l}\text { All surgical instruments used in the case should be covered for transport and returned } \\
\text { for immediate sterilization following case completion. }\end{array}$ \\
\hline 4 & Patient transport to the $\mathrm{OR}$ & $\begin{array}{l}\text { Staff transporting the patient will facilitate positioning patient on OR bed prior to exiting } \\
\text { the room, to minimize the number of people in the room at one time. }\end{array}$ \\
\hline 5 & Surgical staff preparation & $\begin{array}{l}\text { Surgical staff should be limited to essential personnel to avoid fux of multiple profes- } \\
\text { sionals into the room. } \\
\text { Surgeons, scrub technicians/surgical assistants, and nurses MUST double glove when } \\
\text { scrubbed in the case. }\end{array}$ \\
\hline 6 & Anesthesia considerations & $\begin{array}{l}\text { Endotracheal intubations should be performed by the most experienced individual in } \\
\text { the OR setting. } \\
\text { Closed suction systems should be used for airway aspiration and suctioning. }\end{array}$ \\
\hline 7 & Surgical approach & $\begin{array}{l}\text { Surgical approach should be dictated by best-practice accounting for reduced opera- } \\
\text { tive times and optimal surgical outcomes. }\end{array}$ \\
\hline 8 & Case completion & $\begin{array}{l}\text { Following completion of the operative case, patients should recover in the COVID-19 OR } \\
\text { until able to be transported directly to the appropriate unit outside of the OR. }\end{array}$ \\
\hline
\end{tabular}

COVID-19: corona virus disease 2019, OR: operating room. 
blood transfusion. A postoperative brain CT scan showed nearly total evacuation of the intracerebral hematoma in the bone flap removal state and no remnant hemorrhaging (Fig. 3).

The patient recovered consciousness gradually after the operation and was under close monitoring with no deterioration due to the coronavirus. The Table 1 shows changes in the patient's positive levels as indicated by COVID-19 PCR tests.

\section{DISCUSSION}

Various situations in the medical community have arisen with the prolonged COVID-19 pandemic. In particular, as the number of asymptomatic COVID-19 patients increases, emergency surgical treatment for an increasing number of these patients at a regional trauma center is required.

De Simone et al. [3] suggested that all efforts should be made to assess the possibility of delaying surgery until the patient is no longer at risk of infection or complications during surgery. However, in situations where emergency surgery is difficult to avoid due to deterioration of the patient's condition, it is necessary to prepare for surgery by considering the following the recommendations. A series of recommendations from the European Society of Trau- ma and Emergency Surgery (ESTES) regarding perioperative preparation in a hospital during the COVID-19 pandemic were compiled to inform surgeons about how to carry out emergency surgery and post-trauma care during the pandemic (Fig. 4) [4]. Thorough surgical preparation by the medical institution is warranted to protect both the patient and medical workers from contracting infections. The medical institution should closely examine the operation process to maintain the facilities necessary for an operation and plan the placement of human resources and patient movements. Further, specific guidelines for commodity, environmental, and waste management should be established in accordance with the agency's situation, and medical staff should be continuously trained using the established guidelines [5].

There exist many guidelines for wearing personal protective equipment, and the recommendations of the American College of Surgeons are as follows [6-8]. First, a full-body protective suit (including shoe covers), gloves and a powered air-purifying respirator are standard (if unavoidable, a waterproof full-body gown, KF94 or N95 or higher mask, goggles or a face shield, and gloves can be used). Also, medical personnel in the anesthesiology department and the anesthesia recovery room who are involved in patient intubation or extubation are also included. Last, after disinfection of the surgical site, the gloves and gowns should be promptly exchanged before starting

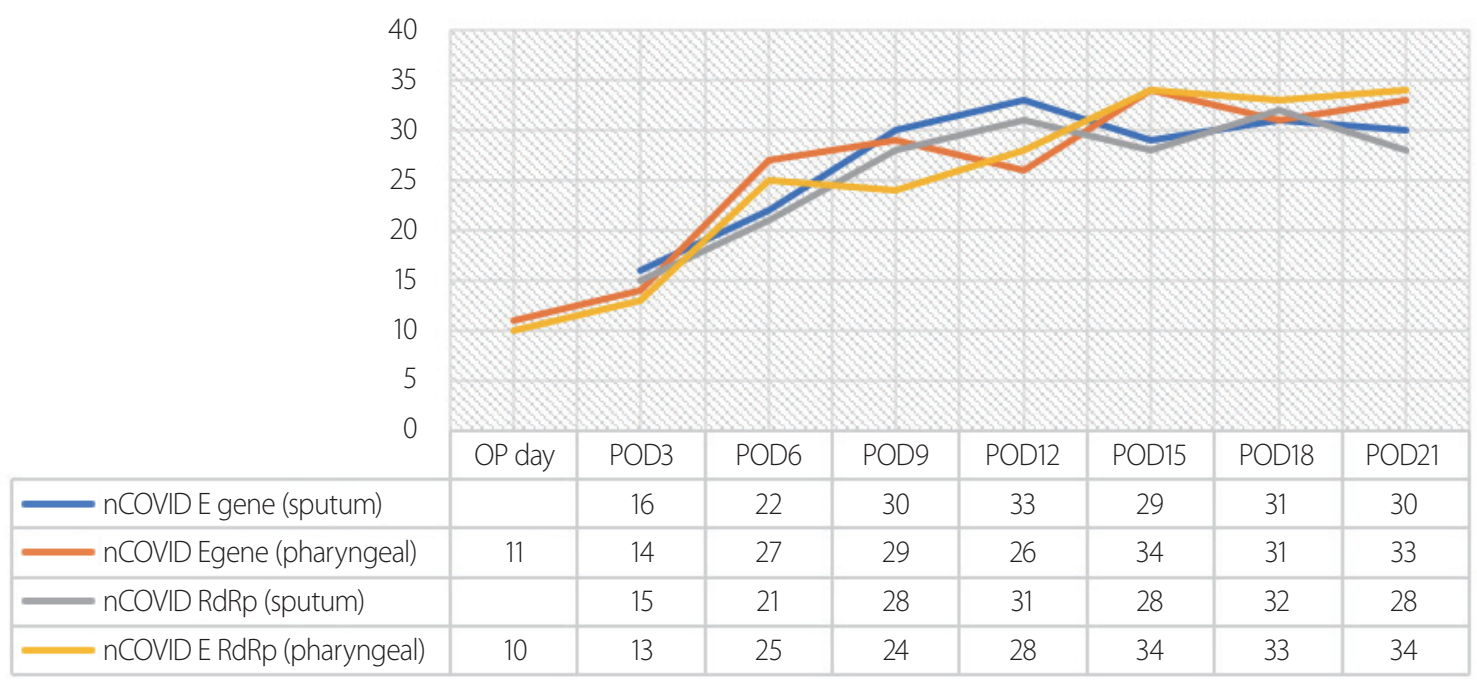

Fig. 4. The patient's COVID-19 PCR test results. OP: opeartion, POD: postoperative day, COVID: coronavirus disease, PCR: polymerase chain reaction. 
surgery. Our team followed a similar protocol and formed a transportation team to transfer patients step by step (Fig. $5)$.

According to the policy of infection control during this pandemic, COVID-19 disease diagnosis should be prioritized in the case of patients admitted for a surgical intracranial lesion. To comply with the objectives, the following should be considered: 1) minimizing exposure in the operating room (OR); 2) decreasing the risk of environmental contamination; 3) minimizing occupation of the OR; and 4) reducing the duration of the hospital stay for patients submitting to surgery [9].
Emergency surgery is an indispensable situation for infected patients, and surgery for traumatic brain injury with hemorrhaging in patients with COVID-19 is currently not studied and reported.

In our experience, there are three main points to consider when treating patients with infectious diseases: first, securing clear movement is necessary. It requires for safety surgery "patient transport team" including at least three to four medical staff, and the movements should be identified in advance so that entry of other patients and medical personnel can be blocked. In addition, the transport team must include certain medical personnel,
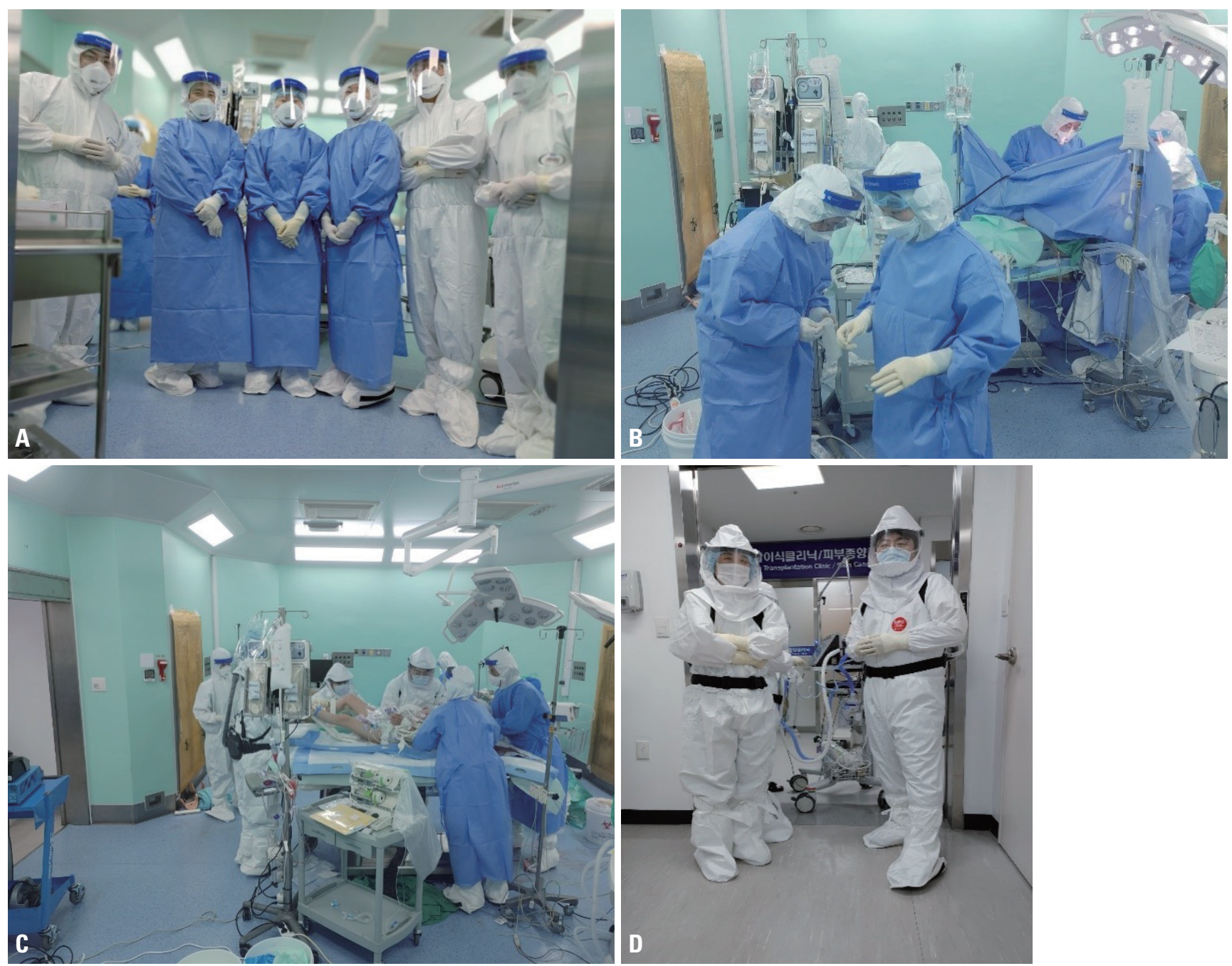

Fig. 5. (A) Coronavirus surgical team consisted of two surgeons, three anesthesiologists, and two surgical assistant nurses. (B) All medical staff participated in the surgery while wearing PPE, and (C, D) the transportation team included an anesthesiologist who could maintain ventilator care and a surgeon who could deal with emergencies during transportation. PPE: personal protective equipment. 
including doctors who can handle emergencies. Second, during surgery, surgeons, the anesthesia team, and the surgical preparation team must be organized and operate as a team. This will minimize fatigue among the medical staff and ensure the best performance when treating the next emergency patient. There are a minimum number of medical resources, including two surgical staff, two surgical assistant nurses, and two or three anesthesia doctors, that must be employed outside the OR. Third, postoperative care is essential for the combined care of both surgical and infectious teams. Patients should be treated in an isolated room for at least 2-3 weeks, and treatment principles should be applied to minimize infection and surgical complications. To do this, the surgical team may need to deviate from the general principles and work out flexible treatment principles. The postoperative patient's consciousness should also be checked with a minimum amount of examinations, including radiologic studies and blood sampling.

Emergency surgery for patients with coronavirus should be performed by well-prepared medical personnel in well-prepared institutions. However, medical staff in trauma centers for emergency patients that cannot be transported to these well-equipped hospital facilities need to be fully prepared for surgical treatment of coronavirus patients using routine preparation and practices.

\section{REFERENCES}

1. Chen N, Zhou M, Dong X, Qu J, Gong F, Han Y, et al. Epidemiological and clinical characteristics of 99 cases of 2019 novel coronavirus pneumonia in Wuhan, China: a descriptive study. Lancet 2020;395:507-13.
2. Wang D, Hu B, Hu C, Zhu F, Liu X, Zhang J, et al. Clinical characteristics of 138 hospitalized patients with 2019 novel coronavirus-infected pneumonia in Wuhan, China. JAMA 2020;323: 1061-9.

3. De Simone B, Chouillard E, Di Saverio S, Pagani L, Sartelli M, Biffl WL, et al. Emergency surgery during the COVID-19 pandemic: what you need to know for practice. Ann R Coll Surg Engl 2020;102:323-32.

4. Coimbra R, Edwards S, Kurihara H, Bass GA, Balogh ZJ, Tilsed J, et al. European Society of Trauma and Emergency Surgery (ESTES) recommendations for trauma and emergency surgery preparation during times of COVID-19 infection. Eur J Trauma Emerg Surg 2020;46:505-10.

5. Han SH, Kim SR, Cha K, Son HJ, Shin MJ, Choi JR, et al. Infection control during surgery for patients with confirmed or suspected coronavirus disease-19. Korean J Healthc Assoc Infect Control Prev 2020;25:4-10.

6. American College of Surgeons. COVID-19: considerations for optimum surgeon protection before, during, and after operation [Internet]. Chicago (IL): American College of Surgeons 2020 [cited 2020 May 30]. Available from: https://www.facs.org/ covid-19/clinical-guidance/surgeon-protection.

7. Ti LK, Ang LS, Foong TW, Ng BSW. What we do when a COVID-19 patient needs an operation: operating room preparation and guidance. Can J Anaesth 2020;67:756-8.

8. Kim JY, Song JY, Yoon YK, Choi SH, Song YG, Kim SR, et al. Middle east respiratory syndrome infection control and prevention guideline for healthcare facilities. Infect Chemother 2015;47:278-302.

9. De Simone B, Chouillard E, Sartelli M, Biffl WL, Di Saverio S, Moore EE, et al. The management of surgical patients in the emergency setting during COVID-19 pandemic: the WSES position paper. World J Emerg Surg 2021;16:14. 